\title{
Produção científica de 10 anos da revista Psicologia Escolar e Educacional (1996/2005)
}

\author{
Katya Luciane Oliveira \\ Lucicleide Maria de Cantalice \\ Maria Cristina Rodrigues Azevedo Joly \\ Acácia Aparecida Angeli dos Santos \\ Universidade São Francisco
}

Resumo

Esta pesquisa visou investigar a produção científica de dez anos de publicações da Psicologia Escolar e Educacional. A análise baseou-se em alguns critérios da metaciência, a saber, autoria, temática, discurso e análise dos tipos de avaliações. Os resultados evidenciaram que a Psicologia Escolar e Educacional aumentou o número de artigos publicados nos últimos cinco volumes, bem como manteve a regularização de sua periodicidade. A maior parte das publicações foi de pesquisas de campo, e a região sudeste foi a que apresentou maior número de trabalhos. Houve predominância da autoria múltipla e feminina. A diversificação das temáticas foi ampliada, havendo uma distribuição entre vários assuntos de interesse para a área. O nível escolar mais estudado foi o ensino superior e o menos focalizado nos artigos foi o ensino médio. Conclui-se que a produção divulgada no período revela o amadurecimento da área, mas aponta para alguns aspectos que poderão ser mais explorados em futuras publicações.

Palavras-chave: Produção científica; Publicações; Psicologia.

\section{The ten-year Scientific production of School and Educational journal (1996/2005)}

\begin{abstract}
This research aimed to investigate the scientific production from the ten-year publication of the "School and Educational Psychology" journal. The analysis was based in some criterias of metascience, knwoing, authorship, thematic, speech and analysis of the types of evaluations. The results evidenced that the journal increased the number of paper publications in the last five volumes, it also keept its periodicy regular. The most part of publications was of field researches, and the SE region was the one that showed the higher number of projects. There were predominance of multiple authorship anf of women. The diversification of the themes was extended along with a distribution between many subjects of interest to the area. The school most studied level was the high school and the one with less focalization in the articles was the $\mathrm{K} 9$ to $\mathrm{KI}$. It was concluded that the divulged production in the the period reveals the matureness of the area, but points out some aspects that might be explored in future publications.
\end{abstract}

Keywords: Scientific productions; Publications; Psycholoy

\section{Producción científica de 10 años de la Psicología Escolar y Educacional (1995/2005)}

\section{Resumen}

Este trabajo buscó investigar la producción científica de diez años de publicaciones de la psicología escolar y educacional. El análisis se asentó en algunos criterios de la meta ciencia, son ellos, autor, tema, discurso y análisis de los tipos de evaluaciones. Los resultados mostraron que la psicología escolar y educacional aumentó el número de artículos publicados en los últimos cinco volúmenes, así como también mantuvo la regularidad de su periodicidad. La mayor parte de las publicaciones fue de investigaciones de campo y la región sureste fue la que presentó el mayor número de trabajos. Hubo una predominancia de trabajos con múltiplos autores y del sexo femenino. La diversidad de temas fue amplia, con una distribución entre varios asuntos de interés para el área. El nivel escolar más estudiado fue la enseñanza superior y el menos tratado en los artículos fue la enseñanza secundaria. Se concluyó que la producción divulgada en el período revela la madurez del área, pero apunta para algunos aspectos que podrán ser más explorados en publicaciones futuras. Palabras-clave: Publicaciones, Psicología, Producción científica. 


\section{Introdução}

O presente estudo tem como finalidade refletir sobre as tendências de publicações realizadas no campo da produção científica em psicologia escolar e educacional. A decisão de se avaliar a produção foi fundamentada no fato de que a revista Psicologia Escolar e Educacional, cuja entidade responsável é a Associação Brasileira de Psicologia Escolar e Educacional (ABRAPEE), comemorou dez anos de publicações da produção intelectual desse campo do saber.

A revista em análise tem por finalidade divulgar o conhecimento de práticas e pesquisas originais e atuais no campo da Psicologia Escolar e Educacional. Ao lado disso, visa a uma ampliação das posições e um intercâmbio entre as diferentes perspectivas de atuação. Portanto, trata-se de um valioso recurso que, por meio da neutralidade e compromisso social, possibilita uma alternativa para aqueles que buscam minimizar as dificuldades que permeiam o contexto escolar (ABRAPEE, 2006).

Com essa perspectiva buscou-se mapear e discutir as diretrizes adotadas para o conhecimento no campo escolar. Também se aventou sobre a importância da ABRAPEE para o avanço científico da área, considerando que a entidade representa de forma legítima o profissional, o pesquisador e o estudante. Cabe lembrar, a título de exemplo, a efetiva participação da ABRAPEE no Congresso Brasileiro de Psicologia de 2000 (Santos, Oliveira, Joly \& Suehiro, 2003).

Witter $(1996,1999)$ aponta que a avaliação da qualidade da produção científica visa ao desenvolvimento da psicologia como campo sólido de investigação. Portanto, analisar a produção científica de uma determinada área do conhecimento psicológico possibilita a compreensão da inserção e impactos causados pela publicação, principalmente quando essa é proveniente de um periódico da área com circulação nacional, como é o caso da revista Psicologia Escolar e Educacional.

Pode-se entender como periódico uma publicação constante que veicula textos científicos, no meio de editoração científica, na Coordenação de
Aperfeiçoamento de Pessoal de Nível Superior e na Fundação de Amparo à Pesquisa do Estado de São Paulo. No Brasil, este termo é usado para diferenciar revista técnica ou de divulgação científica a que ele se refere. Vale ressaltar que a produção científica referese a um processo dinâmico e inacabado, merecedor de constante revisão. É a partir de novas descobertas e de sua divulgação que a ciência é ampliada, as questões são reformuladas e que o conhecimento vai sendo descoberto, analisado e construído (Domingos, 1999).

Nesse contexto, a avaliação da produção científica em psicologia, segundo Freitas (1998), promove o crescimento da área, pois permite o entendimento e o estabelecimento de indicadores de produção e de critérios mais claros e precisos para a concessão de fomento à pesquisa. A avaliação e posterior divulgação da produção científica facilita o acesso ao conhecimento, bem como permite traçar o perfil das pesquisas realizadas sobre temas específicos em determinados períodos (Prat, 1998; Souza Filho, Belo \& Gouveia, 2006). Mais recentemente o acesso a produções em bases de dados online tem permitido que haja uma disseminação muito mais rápida do conhecimento produzido nas diversas áreas do conhecimento.

Dentre as bases de dados, destacam-se o SciELO, a base LILACS (BIREME - Centro Latino Americano e do Caribe de Informação em Ciências da Saúde) e o ISI (Institute for Scientific Informaticon). Mesmo com a democratização da produção, por intermédio das bases de dados, a produção científica brasileira ainda é restrita, especialmente quando se considera o cenário mundial (Meneghini, 1998; Izique, 2002).

Para Carelli (2002) e Bariani, Buin, Barros e Escher (2004) uma provável causa da pouca produção, no caso da área de psicologia, se deve à necessidade de maiores investimentos das agências de fomento à atividade científica. As principais agências que financiam a atividade científica, tais como CAPES, CNPq, FAPESP, dentre outros, necessitam ampliar a demanda de distribuição de bolsas, sobretudo aquelas direcionadas as atividades de iniciação científica.

Nessa direção, Yamamoto, Souza e Yamamoto ( 1999) observam que a avaliação da produção científica é um fato presente no contexto científico internacional. 
Muitas agências de fomento à pesquisa, em diferentes países, têm se empenhado na realização de avaliações da produção, com maior atenção, àquelas publicações em periódicos científicos que apresentam reconhecido rigor e qualidade em seu processo editorial. Todavia, no Brasil há uma carência de estudos cientométricos recorrentes que conceituem e caracterizem a publicação psicológica nacional.

A cientometria prima pela análise da quantidade da produção científica. Tal análise se insere no campo metacientífico (Witter, 1999) que busca avaliar a qualidade do conteúdo publicado. Com a investigação metacientífica é possível caracterizar os indicadores que permeiam essa qualidade, tais como fomento a pesquisa, concentração de publicações por regiões, aspectos metodológicos da pesquisa como discurso, temática e dados, entre outros.

No caso da avaliação da produção científica em psicologia escolar, poucos estudos foram desenvolvidos, visto que essa área é um campo de atuação recente dentro da Psicologia. Tal fato faz com que constantemente estudiosos busquem novos dispositivos para a sua atuação, que, no início, estava vinculada à clínica (Kupfer, 2004).

Com o passar do tempo, pouco a pouco o psicólogo escolar passou a alargar o seu campo de trabalho, abrindo espaço para a circulação de discursos e entendimento das queixas advindas do contexto escolar e educacional. Mas ainda há muito que ser conquistado, principalmente em relação à pesquisa científica, pois segundo Del Prette (1999), há profissionais que desenvolvem suas atividades em situações escolares afastados do campo científico e há os profissionais envolvidos com pesquisas científicas, atuando a produzindo conhecimentos em universidades.

Essa distância entre os próprios profissionais da área de Psicologia Escolar faz com que o conhecimento buscado em produções científicas não chegue ao campo de atuação, impedindo a sua aplicabilidade. Assim, estudiosos apontam em suas investigações essa preocupação, e nota-se um direcionamento dos trabalhos no caminho de intersecção entre teoria e prática (Freitas, 1998; Gomes, 1999; Witter, 1999; Joly, 2000; Almeida, 2002). Portanto, conhecer a quantidade e a qualidade dessa produção possibilita o avanço tão necessário da área.

Witter, Witter, C., Yukimitsu e Gonçalves (1992) realizaram um estudo sobre a atuação do psicólogo escolar e educacional no Brasil, no período de 1980 a 1992 e verificaram que os trabalhos enfatizam mais a prática do que a formação e constataram que se busca ainda uma definição e reconhecimento para a área. Os resultados também revelaram a necessidade de novos estudos acerca do tema.

O estudo de Witter C. (1996), no qual foi realizada a análise de três periódicos nacionais, seis internacionais e dois anais de congressos (um nacional e outro estrangeiro) revelou que a maior produção está relacionada à área da psicologia clínica. $\mathrm{Na}$ área de psicologia escolar as pesquisas realizadas refletem uma produção acerca dos processos psicológicos, disciplinas acadêmicas, aprendizagem e dificuldades no processo de ensino e aprendizagem.

Freitas (1998) realizou um estudo com o objetivo de analisar os procedimentos de avaliação da produção científica no Brasil. Analisou os procedimentos quantitativos e qualitativos, avaliação por pares e avaliações de pesquisas realizadas pelas agências governamentais de fomento. Verificou que a avaliação da produção científica é realizada, porém sem estabelecimento de critérios definidos.

Outro estudo que merece destaque trata-se do trabalho realizado por Santos e cols. (2003), no qual as autoras analisaram a produção científica em Psicologia Escolar e Educacional apresentada em 304 painéis no I Congresso Brasileiro de Psicologia, Ciência e Profissão. Os resultados indicaram diversificação dos temas, um bom nível nos resumos de elaboração quanto ao discurso, predominância de relatos de pesquisa em relação às outras categorias e análise qualitativa dos dados. Além disso, as autoras chamam a atenção para o fato do número de painéis apresentados na área revelando uma boa representatividade do campo de atuação em relação às demais áreas de trabalhos apresentados.

Bariani e cols. (2004) em seu estudo sobre a produção em psicologia escolar no ensino superior, concluiu que as pesquisas, de um modo geral, utilizam o corpo discente ingressante e concluinte. Possuem como 
principal temática o processo de ensino aprendizagem. Há uma carência de estudos experimentais nesse contexto e a predominância de estudos descritivos.

Estudos de produção permitem uma leitura crítica das publicações científicas. Estas, por sua vez, possibilitam a qualificação de possíveis problemas veiculados ao produto: conhecimento. Sendo assim, trabalhos sobre produção científica assumem grande relevância, pois fornecem um mapeamento das contribuições, necessidades e déficits nas diversas áreas do conhecimento. Com base nessas considerações, avaliar a produção científica da revista Psicologia Escolar e Educacional num período de dez anos, foi o objetivo desta pesquisa.

\section{Método}

\section{Fonte}

Foram analisados 21 números da revista Psicologia Escolar e Educacional, totalizando I 36 artigos. Vale acrescentar que a referida revista apresenta um conceito de avaliação 'A' nacional. Utilizou-se versões impressas dos periódicos, bem como versões disponíveis on-line, na página da ABRAPEE.

Constituiu-se como material de análise somente as publicações que se encontravam na modalidade 'Artigos'. Outras formas de publicações não foram foco deste estudo. Estabeleceu-se um período de dez anos (1996-2005) para a realização da análise.

\section{Procedimento}

Nestas publicações buscou-se avaliar os artigos na íntegra. Para tanto, utilizou-se alguns critérios estabelecidos por Witter (1999) para se investigar os volumes da revista. Os itens pesquisados foram a quantidade e a média de volumes publicados por ano; a distribuição do tipo de trabalho (relato de pesquisa, relato de experiência, pesquisa documental, manuscrito teórico); a distribuição da produção por origem (sul, sudeste, norte, nordeste, centro-oeste, parcerias regionais, internacional e parcerias nacionais e internacionais); à natureza da autoria (individual ou múltipla) e o gênero dos autores; a quantidade e a distribuição por temas de avaliação; as palavras contidas no título do trabalho e número e escolaridade dos participantes; a classificação do tipo de instrumentos empregados nas avaliações.

\section{Resultados}

Os dados foram organizados em planilha e submetidos à estatística descritiva. No que se refere à quantidade de volumes números e o total de artigos publicados, a Tabela I apresenta a distribuição das publicações.

Observa-se que os últimos três anos apresentaram uma média maior de publicações, especialmente, o ano de 2005. Contata-se, também, que do ano 2000 até o último volume analisado (2005) houve uma periodicidade nas publicações, isto é, a revista passou a apresentar dois números anuais por volume.

No que tange a análise por tipo de trabalho, buscou-se investigar a modalidade na qual o manuscrito se enquadrava. Para tanto, considerou-se as seguintes categorias: relato de pesquisa de campo, relato de pesquisa documental ou manuscrito teórico. Os dados evidenciaram que a maior parte $(67,7 \%, n=92)$ se referia a relatos de pesquisas de campo, em seguida apareceram os manuscritos teóricos $(22 \%, n=30)$ e, por fim, os relatos de pesquisas documentais $(10,3 \%, n=14)$. Para avaliar se a distribuição da modalidade dos trabalhos era eqüitativa, recorreu-se ao teste Qui-quadrado cujo resultado revelou que a distribuição não era eqüitativa $\left[\chi^{2}(2,136)=74,88\right.$; $p<0,00$ I] .

Para avaliar a origem da produção levantou-se a procedência do artigo. Nesse sentido, avaliou-se a região, o país e parcerias (regionais e internacionais). A Tabela 2 apresenta a distribuição dos artigos por origem.

Novamente o teste Qui-quadrado foi utilizado para levantar se a distribuição da origem dos trabalhos era eqüitativa. A distribuição não foi eqüitativa, considerando $\left[\chi^{2}(6,136)=210,19 ; p d " 0,001\right]$, confirmando o sudeste como o maior pólo de publicações na revista.

No que se refere à autoria dos trabalhos, em razão da realização individual ou múltipla, evidenciou-se que $58,1 \%(n=79)$ eram publicações múltiplas, isto é, que 
Tabela I. Distribuição geral da quantidade de volumes e número, total de artigos publicados e média de publicações $(N=136)$.

\begin{tabular}{|c|c|c|c|c|c|}
\hline Ano & Volumes & Números & $\begin{array}{l}\text { Total de } \\
\text { Artigos por } \\
\text { Número }\end{array}$ & $\begin{array}{c}\text { Total de } \\
\text { Publicações } \\
\text { Ano } \\
\end{array}$ & $\begin{array}{l}M \text { de Publicação } \\
\text { Ano por Número }\end{array}$ \\
\hline 1996 & 1 & 1 & 8 & 8 & 8 \\
\hline 1997 & 1 & 2 e 3 & 6 & 6 & 6 \\
\hline \multirow[t]{3}{*}{1998} & 2 & 1 & 4 & & \\
\hline & & 2 & 8 & 17 & 5,6 \\
\hline & & 3 & 5 & & \\
\hline \multirow[t]{3}{*}{1999} & 3 & 1 & 7 & & \\
\hline & & 2 & 3 & 13 & 4,3 \\
\hline & & 3 & 3 & & \\
\hline \multirow[t]{2}{*}{2000} & 4 & 1 & 3 & 8 & 4 \\
\hline & & 2 & 5 & & \\
\hline \multirow[t]{2}{*}{2001} & 5 & 1 & 6 & 12 & 6 \\
\hline & & 2 & 6 & & \\
\hline \multirow[t]{2}{*}{2002} & 6 & 1 & 7 & 14 & 7 \\
\hline & & 2 & 7 & & \\
\hline \multirow[t]{2}{*}{2003} & 7 & 1 & 9 & 17 & 8,5 \\
\hline & & 2 & 8 & & \\
\hline \multirow[t]{2}{*}{2004} & 8 & 1 & 9 & 18 & 9 \\
\hline & & 2 & 9 & & \\
\hline \multirow[t]{2}{*}{2005} & 9 & 1 & 12 & 23 & 11,5 \\
\hline & & 2 & 11 & & \\
\hline
\end{tabular}

Tabela 2. Distribuição da produção por origem $(n=\mid 36)$.

\begin{tabular}{lcc}
\hline Origem & $F$ & $\%$ \\
\hline Sudeste & 77 & 56,6 \\
Internacional & 20 & 14,7 \\
Sul & 13 & 9,5 \\
Parcerias & 11 & 8,1 \\
Centro-oeste & 9 & 6,6 \\
Nordeste & 5 & 3,7 \\
Norte & 1 & 0,7 \\
\hline
\end{tabular}

envolviam mais de um autor na publicação. A outra parte $(41,9 \%, n=57)$ tratava-se de publicações individuais. O teste Qui-quadrado não mostrou diferença significativa entre a publicação individual ou múltipla $\left[\chi^{2}(I, 136)=3,56 ; p=5,92\right]$.

A análise da autoria por gênero mostrou que $63,9 \%$ $(n=87)$ das publicações eram produções realizadas por mulheres, $24,3 \%(n=33)$ tratavam-se de parcerias entre homens e mulheres e $11,8 \%(n=16)$ eram realizadas por homens. O teste Qui-quadrado apontou uma distribuição não eqüitativa $\left[\chi^{2}(2\right.$,
136) $=60,63 ; p<0,00 \mathrm{I}]$. Esses dados apontam que há um predomínio de publicações nas quais os autores são do gênero feminino.

No que se refere ao título dos trabalhos, foi realizada uma análise considerando a quantidade de palavras contidas nos títulos das pesquisas. Na Tabela 3 pode-se observar tal distribuição.

Houve uma maior concentração de artigos que apresentavam em seus títulos de 8 a 10 palavras. Esse resultado foi confirmado pelo teste Qui-quadrado que mostrou diferença significativa entre as diferentes 
Tabela 3. Análise das palavras contidas no título $(n=136)$.

\begin{tabular}{lcc}
\hline Quantidade de Palavras & $F$ & $\%$ \\
\hline 8 a 10 & 56 & 41,2 \\
11 a 13 & 43 & 31,6 \\
3 a 7 & 24 & 17,6 \\
14 ou mais & 13 & 9,5 \\
\hline
\end{tabular}

categorias, considerando $\left[\chi^{2}(3,136)=32,53\right.$; $p<0,00$ I]

Foi realizada uma análise considerando a distribuição dos manuscritos por temáticas pesquisadas. Desse modo, algumas categorias foram criadas e a Tabela 4 apresenta a freqüência dos trabalhos por temas agrupados.
Assim sendo, observa-se que há assuntos ainda muito pouco pesquisados na literatura nacional, especialmente quando se considera a vasta produção sobre esses temas no cenário internacional, como observou Witter, C. (1996).

Uma análise dos participantes nas pesquisas também foi realizada, ainda considerando os 92 artigos

Tabela 4. Distribuição das temáticas pesquisadas $(n=\mid 36)$.

\begin{tabular}{lcc}
\hline Temáticas & $F$ & $\%$ \\
\hline Testes/construção de instrumentos & 14 & 10,4 \\
Método de ensino/aprendizagem & 13 & 9,5 \\
Leitura & 11 & 8,1 \\
Criatividade & 11 & 8,1 \\
Aspectos afetivos/emocionais & 10 & 7,3 \\
Inteligência/raciocínio/cognição & 9 & 6,6 \\
Psicologia escolar & 9 & 6,6 \\
Relação família/escola & 8 & 5,9 \\
Dificuldades/distúrbios de aprendizagem & 6 & 4,4 \\
Sociabilidade & 6 & 4,4 \\
Desempenho acadêmico & 6 & 4,4 \\
Leitura e escrita & 5 & 3,7 \\
Programas educativos & 4 & 2,9 \\
Produção científica & 4 & 2,9 \\
Motivação & 4 & 2,9 \\
Relação professor/aluno & 3 & 2,2 \\
Escrita & 3 & 2,2 \\
Saúde na escola & 2 & 1,5 \\
Estratégias de aprendizagem & 2 & 1,5 \\
Personalidade & 2 & 1,5 \\
Outros & 2 & 1,5 \\
Condições estudo & 1 & 0,7 \\
Orientação Profíssional & 1 & 0,7 \\
\hline
\end{tabular}

A distribuição da produção, considerando apenas as que tiveram seis ou mais trabalhos, mostrou que não houve diferença significativa entre as temáticas pesquisadas.. Utilizando-se o Qui-quadrado obtevese como resultado $\left[\chi^{2}(10,136)=6,61 ; p=67,78\right]$. mencionados como os de pesquisa de campo. $\mathrm{Na}$ Tabela 5 pode ser vista a freqüência e porcentagem do número de participantes nos estudos.

A maior parte dos estudos trabalhou com amostras que variam de 50 a 300 sujeitos. $O$ teste Qui-quadrado 
Tabela 5. Análise do número de participantes/ sujeitos nas pesquisas $(n=92)$.

\begin{tabular}{lcc}
\hline $\begin{array}{l}\text { Categorias do número } \\
\text { de participantes }\end{array}$ & $F$ & $\%$ \\
\hline De 101 a 200 & 14 & 15,2 \\
De 51 a 100 & 13 & 14,1 \\
De 21 a 30 & 11 & 11,9 \\
De 201 a 300 & 11 & 11,9 \\
De 31 a 50 & 10 & 10,9 \\
De 1 a 10 & 8 & 8,8 \\
De 11 a 20 & 7 & 7,6 \\
De 301 a 400 & 5 & 5,4 \\
De 401 a 500 & 4 & 4,3 \\
De 701 a 1000 & 4 & 4,3 \\
Mais de 1000 & 2 & 2,2 \\
Indefinido & 2 & 2,2 \\
De 501 a 700 & 1 & 1,1 \\
\hline
\end{tabular}

não indicou diferença significativa entre o número de participantes na pesquisa $\left[\chi^{2}(11,92)=16 ; 76 p=5,25\right]$.

Avaliou-se a distribuição dos artigos considerando o nível de escolaridade dos participantes dos estudos. $\mathrm{Na}$ Tabela 6 aparecem os percentuais relativos à freqüência de pesquisas focalizando aqueles participantes.

\section{Discussão}

O fato dos últimos anos apresentarem maior média de publicações, bem como uma periodicidade constante em seus números demonstra que houve um aumento crescente das publicações no periódico.

Tabela 6. Distribuição dos trabalhos em razão da etapa de escolarização dos participantes $(n=92)$.

\begin{tabular}{lcc}
\hline Tipos de Avaliação & $F$ & $\%$ \\
\hline Ensino superior & 30 & 32,6 \\
Escolaridade indefinida & 21 & 22,8 \\
Escolaridade mista & 14 & 15,2 \\
Ensino fundamental (de $1^{\mathrm{a}}$ a $4^{\mathrm{a}}$ série) & 12 & 13 \\
Ensino fundamental (geral) & 5 & 5,4 \\
Pré-escola & 4 & 4,3 \\
Ensino fundamental (de $5^{\mathrm{a}}$ a $8^{\mathrm{a}}$ série) & 3 & 3,3 \\
Ensino médio & 2 & 2,2 \\
Jardim da infância & 1 & 1,1 \\
\hline
\end{tabular}

É possível observar-se que o ensino superior foi o nível mais pesquisado, contudo em um número considerável de artigos não foi possível identificar o nível de escolaridade dos participantes. A distribuição das freqüências nas diversas etapas pesquisadas não foi eqüitativa, conforme apontou o teste Qui-quadrado $\left[\chi^{2}(8,92)=77,83 ; p d " 0,001\right]$.
Isso pode estar relacionado à avaliação Qualis da revista a qual possibilitou uma maior demanda de submissões de manuscritos por se tratar de um periódico Nacional A. Esse dado também pode ser interpretado considerando-se que parece haver uma preocupação maior das agências de fomento nos últimos cinco anos em ampliar o investimento em 
pesquisas, o que, segundo Carelli (2002) e Bariani e cols. (2004), indiretamente promove o aumento da produção científica na área.

No que tange à modalidade de publicação, a maior parte se referia a relatos de pesquisa de campo, seguida de manuscritos que traziam considerações teóricas acerca da área escolar. Witter (2003) constata que os estudos na área escolar, de um modo geral, são descritivos, haja vista o baixo número de pesquisas de cunho experimental ou quase-experimental. Tais resultados também corroboram os dados da pesquisa de Santos e cols. (2003) que também apontaram para um predomínio de pesquisas descritivas de campo em relação a outras modalidades de pesquisa e confirmam a tendência destacada por Witter (2003). Em razão disso, parece possível aventar-se que os estudos brasileiros ainda estão muito empenhados em caracterizar os contextos educacionais, sendo importante que se alie cada vez mais o rigor da pesquisa científica acadêmica à prática do cotidiano escolar e de aprendizagem.

A região sudeste despontou como a mais produtiva em termos de publicações na Psicologia Escolar e Educacional. Este resultado é congruente com o de outros autores que já apontaram para o acúmulo de produção científica nessa região (Meneghini, 1998; Souza Filho, Belo \& Gouveia, 2006), que concentra a maior parte dos cursos de psicologia do país, tanto na graduação como na pós-graduação (Yamamoto, Sousa \& Yamamoto, 1999).

Outro aspecto a ser comentado diz respeito ao fato da publicação internacional aparecer como a segunda categoria, em relação ao local de publicação, que mais contribuiu com publicações na revista Psicologia Escolar e Educacional. Com base neste resultado pode-se conjecturar sobre possíveis fatores responsáveis por esse fenômeno, tais como, o compromisso de divulgar o mais amplamente a área escolar/educacional que se observou no aumento crescente das publicações, como também a seriedade da Psicologia Escolar e Educacional enquanto periódico científico revelada pela sua classificação Qualis. Ao lado disso, supõe-se também que a maior visibilidade dada às publicações por sua presença em repositórios virtuais como o PePsic e a página da ABRAPEE também têm atraído colaboradores internacionais.
Quanto à autoria dos trabalhos, a autoria múltipla e a feminina foram às categorias que apresentaram mais publicações na área. Essas constatações não surpreendem visto que é comum e incentivado pelas agências de fomento por meio das características de seus editais a parceria entre vários autores, inclusive de diferentes universidades, para se realizar e publicar uma pesquisa. No caso da autoria feminina, a discussão se insere num âmbito maior, no qual se deve considerar a psicologia como uma profissão que, historicamente tem o predomínio de mulheres; no caso específico da psicologia escolar essa realidade não é diferente.

No tocante ao número de palavras do título dos artigos, evidenciou-se que a maioria tinha de oito a treze palavras. Tal número está adequado às normas científicas que preconizam de oito a doze palavras em um título (Witter, 1996).

A análise do tema indicou freqüência maior de pesquisas em algumas áreas, tais como a de testes/ construção de instrumento, método de ensino e aprendizagem, leitura e criatividade, bem como sobre os aspectos afetivos/emocionais. Chama atenção o fato de que a orientação profissional, assunto tão relevante em vários contextos educacionais tenha apresentado apenas uma publicação. Uma explicação possível seria a que os estudiosos desse tema têm no Brasil um lócus próprio de publicação, que é um periódico específico da Associação Brasileira de Orientação Profissional (ABOP), mas mesmo com assim seria esperado que o tema ocupasse um espaço maior na área educacional.

Ainda em busca de explicações para a freqüência de publicação em determinados temas, é possível pressupor que a quantidade de artigos que focalizaram o tema de testes no contexto escolar se justifica, principalmente quando se leva em consideração o crescimento da área de avaliação psicológica no Brasil durante os cinco últimos anos do período pesquisado, também apontado por Souza Filho, Belo e Gouveia (2006). Essa ênfase pode ser resultante do impacto da Resolução $n^{\circ} 25 / 200$ I do Conselho Federal de Psicologia, que definiu padrões para utilização de testes psicológicos no Brasil. Sob essa perspectiva, muitas pesquisas foram realizadas com o objetivo de demonstrar evidências de validade de testes e outras medidas psicológicas, o que repercutiu também no 
aumento das publicações sobre o tema, mesmo considerando que há também um periódico específico da área publicado pelo Instituto Brasileiro de Avaliação Psicológica (IBAP).

Com relação ao número de sujeitos, observou-se que na maior parte das pesquisas relatadas houve uma variação no tamanho da amostra de cinqüenta a trezentos participantes e o nível escolar mais pesquisado foi o ensino superior. Ao que parece estudos com amostras muito pequenas não são priorizados e também há que se considerar que o contexto escolar oferece uma maior possibilidade de participação de um grande número de sujeitos, tendo em vista que se trata de um cenário composto por muitos alunos e docentes, sendo mais fácil lidar com a recusa ou a desistência de participação. No caso do ensino superior é uma área que tem sido utilizada como contexto para diversos estudos por oferecer uma amostra por conveniência. Nesse sentido, as publicações da Psicologia Escolar e Educacional, em sua maioria, trabalharam com essa população. Esse fato remete ao questionamento de que há necessidade de se ampliar os estudos para os outros níveis formais de ensino, principalmente a educação infantil, o ensino fundamental de $5^{\mathrm{a}}$ a $8^{\mathrm{a}}$ séries e, em especial, com o ensino médio que aparece como a etapa menos pesquisada.

\section{Considerações Finais}

Embora a Psicologia Escolar seja uma área de atuação e especialidade da psicologia assim definida pelo Conselho Federal de Psicologia, percebe-se que algumas etapas do ensino formal não estão sendo pesquisadas, mais especificamente o ensino médio e a educação infantil, merecendo maior atenção. Além disso, nota-se uma certa distância entre teoria e prática, uma vez que há predominância de pesquisas sendo realizadas dentro dos contextos de formação acadêmica, enquanto pouco se pesquisa na realidade escolar ou em outros contextos educacionais e de aprendizagem.

Diante disso, a falta de uma articulação entre o discurso teórico e as práticas vivenciadas pelos profissionais pode tornar o trabalho do psicólogo escolar de certa forma fragmentado ou implicar em resistência por parte de alguns profissionais que também atuam na escola. É comum relatos de psicólogos escolares que não têm seu trabalho reconhecido nesse contexto, havendo ainda uma prática clínica na escola sendo confundida com o fazer do psicólogo escolar. Isso ocorre a despeito do reconhecimento do Conselho Federal de Psicologia ao considerar a Psicologia Escolar e Educacional como área de especialidade.

Em suma, vislumbra-se uma perspectiva positiva em relação à consolidação da atuação do psicólogo na escola. Espera-se que as indicações destacadas nesse manuscrito possam servir de indicadores para futuras investigações e publicações, cumprindo assim seu objetivo maior. Isto posto, ressalta-se que a análise ora realizada refere-se a uma área da Psicologia bastante vasta e aberta às diversas possibilidades de pesquisa e atuação.

\section{Referências}

Almeida, L. S. (2002). Facilitar a aprendizagem: ajudar os alunos a aprender e a pensar. Psicologia Escolar e Educacional, 6, I55-165.

Associação Brasileira de Psicologia Escolar e Educacional ABRAPEE. (2006).[online]. Disponível em <http.www.abrapee.psc.br > Acessado em 21 / I l/ 2006.

Bariani, I. C. D., Buin, E., Barros, R. C. \& Escher, C. A. (2004). Psicologia escolar educacional no ensino superior: análise da produção científica. Psicologia Escolar e Educacional, 8, 17-27.

Buriti, M. A. (1999). Produção científica em periódicos de psicologia do esporte e educação fisica. Tese de doutorado. Instituto de Psicologia e Fonoaudiologia da Pontifícia Universidade Católica de Campinas, Campinas.

Carelli, A. E. (2002). Produção científica em leitura: dissertações e teses (1990-1999). Tese de Doutorado. Centro de Ciências da vida. Pontifícia Universidade Católica de Campinas, Campinas.

Conselho Federal de Psicologia - CFP (2001). Resolução $n^{\circ}$ 25/200I. [online] Disponível em <http.:// www.pol.org.br $>$. Acessado em 04/07/2006. 
Del Prette, Z. A. (1999). Psicologia, educação e LDB: novos desafios para velhas questões? Em R. S. L. Guzzo (Org.). Psicologia escolar: LDB e educação hoje (pp. II-34). Campinas,SP: Alínea.

Domingos, N. A. M. (1999). Produção científica: análise de resumos de dissertações e teses em Psicologia (1992/1996). Tese de doutorado. Instituto de Psicologia e Fonoaudiologia da Pontifícia Universidade Católica de Campinas, Campinas.

Freitas, M. H. A. (1998). Avaliação da produção científica: considerações sobre alguns critérios. Psicologia Escolar e Educacional, 2, 21 I-228.

Gomes, V. L. T. (1999). A formação do psicólogo escolar e os impasses entre a teoria e a prática. Em R. S. L. Guzzo (Org.) Psicologia Escolar: LDB educação hoje (pp. 49-73). Campinas <SP: Alínea.

Izique, C. (2002). Produção crescente. Pesquisa FAPESP, 8I, I8-22.

Joly, M. C. R. A. (2000). A formação do Psicólogo Escolar e a educação no terceiro milênio. Psicologia Escolar e Educacional, 4, 5I-55.

Kupfer, M. C. M. (2004). O que toca à/a Psicologia Escolar. Em A. M. Machado \& M. P. R. Souza (Orgs.) Psicologia escolar: em busca de novos rumos (55-65). São Paulo <SP: Casa do Psicólogo.

Meneghini, R. (1998). Avaliação da produção científica e o projeto SciELO. Ciência da Informação, 27(2). [on line] Disponível em < http: www.scielo.br> Acessado em 28/09/2005.

Prat, A. M. (1998). Avaliação da produção científica como instrumento para o desenvolvimento da ciência e da tecnologia. Ciência da Informação, 27(2), [on line] Disponível em < http: www.scielo.br> Acessado em 28/09/2005.
Rudio, F. V. (1985). Introdução ao projeto de pesquisa. Petrópolis,RJ: Vozes

Santos, A. A. A., Oliveira, K. L. Joly, M. C. A. \& Suehiro, A. C. B. (2003). Produção científica da área de escolar no I Congresso Brasileiro de Psicologia Ciência e Profissão. Psicologia Escolar e Educacional, 7, I35-144.

Souza Filho, M. L., Belo, R. \& Gouveia, V. V. (2006) Testes psicológicos: análise da produção científica brasileira no período de 2000-2004. Psicologia: Ciência e Profissão, 26, 478-489.

Yamamoto, O. H., Souza, C. de Souza \& Yamamoto, M. E. (1999). A produção científica na psicologia: uma análise dos periódicos brasileiros no período de 1990-1997. Psicologia: Reflexão e Crítica, 12, 549-565.

Witter, C. (1996). Psicologia Escolar: produção científica, formação e atuação (1990-1994). Tese de doutorado. Universidade de São Paulo. São Paulo.

Witter, G., Witter, C.; Yukimitsu, M. T. C. P. \& Gonçalves, C. L. C. (1992). Atuação do psicólogo escolar no Brasil; perspectivas através de textos (1980-1992). Em Conselho Federal de Psicologia. Psicólogo Brasileiro: construção de novos espaços (pp. 23-53). Campinas, SP: Átomo.

Witter, G. P. (1999). Metaciência e leitura. Em G. P.Witter (Org.), Leitura: textos e pesquisas (pp. 13-22). Campinas,SP: Alínea.

Witter, G. (2003). Professor - estresse. Psicologia Escolar e Educacional, 7, 33-46.

Recebido em: 23/08/2006

Revisado em: 06/10/2006

Aprovado em: 19/12/2006

Sobre as autoras

Katya Luciane Oliveira (katya_lincoln@ig.com.br) é doutoranda em Psicologia e docente do curso de Psicologia da Universidade São Francisco

Lucicleide Maria de Cantalice (lucicleide.cantalice@saofrancisco.edu.br) é mestre em Psicologia e docente da Universidade São Francisco

Maria Cristina Rodrigues Azevedo Joly (cristina.joly@saofrancisco.edu.br) é Doutora em Psicologia/ docente do programa de pós-graduação stricto sensu da Universidade São Francisco

Acácia Aparecida Angeli dos Santos (acacia.santos@saofrancisco.edu.br) é Doutora em Psicologia/ docente do programa de pós-graduação stricto sensu da Universidade São Francisco

Endereço para correspondência

Katya Luciane Oliveira

Rua. Alexandre Rodrigues Barbosa, 45

|325|-900 - Itatiba, SP

292 Produção científica de 10 anos da revista psicologia escolar e educacional • Katya Oliveira, Lucicleide Cantalice, Maria Cristina Joly e Acácia dos Santos 